Research Article

Open Access

Behzad Djafari Rouhani* and Mohsen Rahimi Piranfar

\title{
Nonhomogeneous nonlinear oscillator with damping: asymptotic analysis in continuous and discrete time
}

https://doi.org/10.1515/dema-2019-0028

Received March 18, 2019; accepted August 3, 2019

Abstract: We consider the following second order evolution equation modelling a nonlinear oscillator with damping

$$
\ddot{u}(t)+\gamma \dot{u}(t)+A u(t)=f(t),
$$

where $A$ is a maximal monotone and $\alpha$-inverse strongly monotone operator in a real Hilbert space $H$. With suitable assumptions on $\gamma$ and $f(t)$ we show that $A^{-1}(0) \neq \emptyset$, if and only if (SEE) has a bounded solution and in this case we provide approximation results for elements of $A^{-1}(0)$ by proving weak and strong convergence theorems for solutions to (SEE) showing that the limit belongs to $A^{-1}(0)$. As a discrete version of (SEE), we consider the following second order difference equation

$$
u_{n+1}-u_{n}-\alpha_{n}\left(u_{n}-u_{n-1}\right)+\lambda_{n} A u_{n+1} \ni f(t),
$$

where $A$ is assumed to be only maximal monotone (possibly multivalued). By using the results in [Djafari Rouhani B., Khatibzadeh H., On the proximal point algorithm, J. Optim. Theory Appl., 2008, 137, 411-417], we prove ergodic, weak and strong convergence theorems for the sequence $u_{n}$, and show that the limit is the asymptotic center of $u_{n}$ and belongs to $A^{-1}(0)$. This again shows that $A^{-1}(0) \neq \emptyset$ if and only if $u_{n}$ is bounded. Also these results solve an open problem raised in [Alvarez F., Attouch H., An inertial proximal method for maximal monotone operators via dicretization of a nonlinear oscillator with damping, Set Valued Anal., 2001, 9, 3-11], namely the study of the convergence results for the inexact inertial proximal algorithm. Our paper is motivated by the previous results in [Djafari Rouhani B., Asymptotic behaviour of quasi-autonomous dissipative systems in Hilbert spaces, J. Math. Anal. Appl., 1990, 147, 465-476; Djafari Rouhani B., Asymptotic behaviour of almost nonexpansive sequences in a Hilbert space, J. Math. Anal. Appl., 1990, 151, 226-235; Djafari Rouhani B., Khatibzadeh H., Asymptotic behavior of bounded solutions to some second order evolution systems, Rocky Mountain J. Math., 2010, 40, 1289-1311; Djafari Rouhani B., Khatibzadeh H., A strong convergence theorem for solutions to a nonhomogeneous second order evolution equation, J. Math. Anal. Appl., 2010, 363, 648-654; Djafari Rouhani B., Khatibzadeh H., Asymptotic behavior of bounded solutions to a class of second order nonhomogeneous evolution equations, Nonlinear Anal., 2009, 70, 4369-4376; Djafari Rouhani B., Khatibzadeh H., On the proximal point algorithm, J. Optim. Theory Appl., 2008, 137, 411-417] and significantly improves upon the results of [Attouch H., Maingé P. E., Asymptotic behavior of second-order dissipative evolution equations combining potential with non-potential effects, ESAIM Control Optim. Calc. Var., 2011, 17(3), 836-857], and [Alvarez F., Attouch H., An inertial proximal method for maximal monotone operators via dicretization of a nonlinear oscillator with damping, Set Valued Anal., 2001, 9, 3-11].

Keywords: nonlinear oscillator with damping, inexact inertial proximal method, asymptotic behavior, maximal monotone operator, asymptotic center

MSC: Primary 35B40, 47H05; Secondary 34C12, 34G20, 34G25

\footnotetext{
*Corresponding Author: Behzad Djafari Rouhani: Department of Mathematical Sciences, University of Texas at El Paso, 500 W. University Ave., El Paso, TX 79968, USA; ORCID 0000-0001-8645-8773; E-mail: behzad@utep.edu Mohsen Rahimi Piranfar: Department of Mathematical Sciences, University of Texas at El Paso, 500 W. University Ave., El Paso, TX 79968, USA; Department of Mathematics, Institute for Advanced Studies in Basic Sciences, P.O. Box 45195-1159, Zanjan, Iran; E-mail: mrahimipira@utep.edu
} 


\section{Introduction}

Let $H$ be a real Hilbert space with scalar product $\langle\cdot, \cdot\rangle$, norm $\|\cdot\|$. We denote weak convergence in $H$ by $\rightarrow$ and strong convergence by $\rightarrow$. An operator $A: D(A) \subset H \rightarrow H$ (possibly multivalued) is called monotone (respectively strongly monotone) if $\left\langle y_{2}-y_{1}, x_{2}-x_{1}\right\rangle \geq 0$, (respectively $\left\langle y_{2}-y_{1}, x_{2}-x_{1}\right\rangle \geq \alpha\left\|x_{2}-x_{1}\right\|^{2}$ for some $\alpha>0$ ) for all $x_{i} \in D(A), y_{i} \in A\left(x_{i}\right)$, for $i=1$, 2. A monotone operator $A$ is maximal if $R(I+A)=H$, where $I$ is the identity operator on $H$. For $\alpha>0$, we call a maximal monotone operator $A: H \rightarrow H \alpha$-inverse strongly monotone, if for all $x$ and $y$ in $H$, we have

$$
\alpha\|A x-A y\|^{2} \leq\langle A x-A y, x-y\rangle .
$$

Obviously, every $\alpha$-inverse strongly monotone operator is single-valued and Lipschitz with Lipschitz constant $\frac{1}{\alpha}$. We denote the first and the second order derivatives of a curve $u$ respectively by $\dot{u}$ and $\ddot{u}$. By introducing the notion of almost nonexpansive sequences and curves in $H$, the asymptotic behavior of solutions to the first order evolution equation

$$
\left\{\begin{array}{l}
\dot{u}(t)+A u(t) \ni f(t), \\
u(0)=u_{0}
\end{array}\right.
$$

where $A$ is a maximal monotone operator in $H$, was studied by Djafari Rouhani $[1,2]$ without assuming the zero set of $A$ to be nonempty. The asymptotic behavior of the dynamical system:

$$
\left\{\begin{array}{l}
\ddot{u}(t)+\gamma \dot{u}(t) \in A u(t)+f(t), \\
u(0)=u_{0}, \sup _{t \geq 0}\|u(t)\|<+\infty,
\end{array}\right.
$$

was studied by Djafari Rouhani and Khatibzadeh [3-5], where $A$ is a maximal monotone operator in $H$ and $\gamma \in \mathbb{R}$. In [6], Alvarez studied the initial value problem

$$
\left\{\begin{array}{l}
\ddot{u}(t)+\gamma \dot{u}(t)+\nabla \phi u(t)=0, \\
u(0)=u_{0}, \quad \dot{u}(0)=u_{1},
\end{array}\right.
$$

where $\gamma>0$ and $\phi: H \rightarrow \mathbb{R}$ is differentiable. This system is called the Heavy Ball with Friction, (HBF) for short, which is a nonlinear oscillator with damping. Assuming that $\phi$ is convex and $\operatorname{Argmin} \phi \neq \emptyset$, he proved that each trajectory of the system (HBF) converges weakly to some minimum point of $\phi$, see $[6,7]$. In 2011, Attouch and Maingé [8] considered (HBF) when $\nabla \phi$ is replaced by $A=\nabla \phi+B$, where $\phi: H \rightarrow \mathbb{R}$ is a convex and continuously differentiable function and $B$ is a maximal monotone operator which is also $\alpha$-inverse strongly monotone for some $\alpha>0$ with $\alpha \gamma^{2}>1$. Assuming $A^{-1}(0) \neq \emptyset$, they obtained the weak convergence of the solution to some point in $A^{-1}(0)$. In the second section of this paper motivated by our previous results [1-5] and by the results in [6-8], we study the asymptotic behavior of the solutions to the following second order evolution equation

$$
\left\{\begin{array}{l}
\ddot{u}(t)+\gamma \dot{u}(t)+A u(t)=f(t), \\
u(0)=u_{0}, \quad \sup _{t \geq 0}\|u(t)\|<+\infty,
\end{array}\right.
$$

where $A$ is an $\alpha$-inverse strongly monotone operator. More precisely, we show weak and strong convergence of $u(t)$ to an element of $A^{-1}(0)$, which is also the asymptotic center of $u(t)$, if and only if $\sup _{t \geq 0}\|u(t)\|<$ $+\infty$, therefore showing that $A^{-1}(0)$ is nonempty if and only if $\sup _{t \geq 0}\|u(t)\|<+\infty$. We note that in [6-8], the existence of bounded solutions was proved by assuming that $A^{-1}(0)$ is nonempty. Here we show that this assumption follows from the existence of bounded solutions. In Section 3, we consider the discrete version of (1.1), which was studied by Alvarez and Attouch [9] for the homogeneous case, and leads to the following inexact inertial proximal algorithm

$$
u_{n+1}-u_{n}-\alpha_{n}\left(u_{n}-u_{n-1}\right)+\lambda_{n} A u_{n+1} \ni f_{n},
$$

where $A$ is a general maximal monotone operator. We prove ergodic, as well as convergence theorems for bounded solutions to (1.2) without assuming $A^{-1}(0) \neq \emptyset$, and provide an answer to the open problem raised in [9]. 
Definition 1.1. Given a bounded curve $u(t)$ in $H$, the asymptotic center $c$ of $u(t)$ is defined as follows (see [10]): for every $q \in H$, let $\phi(q)=\lim \sup _{t \rightarrow+\infty}\|u(t) q\|^{2}$. Then $\phi$ is a continuous and strictly convex function on $H$, satisfying $\phi(q) \rightarrow+\infty$ as $\|q\| \rightarrow+\infty$. Therefore $\phi$ achieves its minimum on $H$ at a unique point $c$, called the asymptotic center of the curve $u(t)$.

For a bounded sequence $u_{n}$ in $H$, its asymptotic center is defined in a similar way.

\section{Nonlinear oscillator with damping}

In this section, we study the asymptotic behavior of the solutions to (1.1). We start by recalling the following lemma from [7]:

Lemma 2.1. Let $h \in C^{1}\left(0,+\infty ; \mathbb{R}^{+}\right)$satisfy the following differential inequality

$$
\ddot{h}(t)+\gamma \dot{h}(t) \leq g(t)
$$

with $g \in L^{1}\left(0,+\infty ; \mathbb{R}^{+}\right)$. Then, $[\dot{h}]_{+}$the positive part of $\dot{h}$ belongs to $L^{1}(0,+\infty ; \mathbb{R})$ and, as a consequence, $\lim _{t \rightarrow+\infty} h(t)$ exists.

The following theorem establishes the weak convergence of the solutions to (1.1).

Theorem 2.2. Let $A$ be $\alpha$-inverse strongly monotone, $f \in L^{1}(0,+\infty ; H)$ and $u(t)$ satisfy (1.1). If $\alpha \gamma^{2}>1$, then $u(t)$ converges weakly to some $p \in A^{-1}(0)$, which is also the asymptotic center of $u(t)$, if and only if $u(t)$ is bounded.

Proof. Necessity is clear. To prove the sufficiency, first we show that $\dot{u}(t)$ is bounded. To this aim, multiplying (1.1) by $e^{\gamma t}$ and then integrating on the interval [0, $\left.t\right]$, we get:

$$
\dot{u}(t)=\dot{u}(0) e^{-\gamma t}-\int_{0}^{t} e^{-\gamma(t-\tau)} A u(\tau) d \tau+\int_{0}^{t} e^{-\gamma(t-\tau)} f(\tau) d \tau .
$$

Since $A$ is Lipschitz, $A u(t)$ is bounded, and therefore $\dot{u}$ is bounded too. Let

$$
w(t):=\frac{1}{t} \int_{0}^{t} u(\tau) d \tau
$$

Since $u(t)$ is bounded, $w(t)$ has a weak cluster point, say $p$. Let $t_{k}$ be a sequence such that $t_{k} \rightarrow+\infty$ and $w\left(t_{k}\right) \rightarrow p$ as $k \rightarrow+\infty$. By the $\alpha$-inverse strong monotonicity of $A$ we have

$$
\begin{aligned}
\langle\ddot{u}(t)-\ddot{u}(s), u(t)-u(s)\rangle+\gamma\langle\dot{u}(t)-\dot{u}(s), u(t)-u(s)\rangle & =-\langle A u(t)-A u(s), u(t)-u(s)\rangle+\langle f(t)-f(s), u(t)-u(s)\rangle \\
& \leq-\alpha\|A u(t)-A u(s)\|^{2}+\langle f(t)-f(s), u(t)-u(s)\rangle .
\end{aligned}
$$

Integrating both sides of the inequality with respect to $s$ on the interval $\left[0, t_{k}\right]$, and then dividing by $t_{k}$, we get:

$$
\begin{aligned}
\langle\ddot{u}(t), u(t) & \left.-\frac{1}{t_{k}} \int_{0}^{t_{k}} u(s) d s\right\rangle-\frac{1}{t_{k}}\left\langle\dot{u}\left(t_{k}\right)-\dot{u}(0), u(t)\right\rangle+\frac{1}{t_{k}}\left(\left\langle\dot{u}\left(t_{k}\right), u\left(t_{k}\right)\right\rangle-\langle\dot{u}(0), u(0)\rangle\right)-\frac{1}{t_{k}} \int_{0}^{t_{k}}\|\dot{u}(s)\|^{2} d s \\
& +\gamma\left\langle\dot{u}(t), u(t)-\frac{1}{t_{k}} \int_{0}^{t_{k}} u(s) d s\right\rangle-\frac{\gamma}{t_{k}}\left\langle u\left(t_{k}\right)-u(0), u(t)\right\rangle+\frac{\gamma}{2 t_{k}}\left(\left\|u\left(t_{k}\right)\right\|^{2}-\|u(0)\|^{2}\right) \\
\leq & -\frac{\alpha}{t_{k}} \int_{0}^{t_{k}}\|A u(t)-A u(s)\|^{2} d s+\left\langle f(t), u(t)-\frac{1}{t_{k}} \int_{0}^{t_{k}} u(s) d s\right\rangle-\frac{1}{t_{k}}\left\langle\int_{0}^{t_{k}} f(s) d s, u(t)\right\rangle+\frac{M_{1}}{t_{k}} \int_{0}^{t_{k}}\|f(s)\| d s,
\end{aligned}
$$


where $M_{1}:=\sup _{t \geq 0}\|u(t)\|$. Taking the lim sup as $k \rightarrow+\infty$ in the above inequality, we get:

$$
-\langle A u(t), u(t)-p\rangle \leq \limsup _{k \rightarrow+\infty} \frac{1}{t_{k}} \int_{0}^{t_{k}}\left(\|\dot{u}(s)\|^{2}-\alpha\|A u(t)-A u(s)\|^{2}\right) d s .
$$

On the other hand

$$
\begin{aligned}
\|A u(t)-A u(s)\|^{2}= & \|A u(t)+\ddot{u}(s)+\gamma \dot{u}(s)-f(s)\|^{2} \\
= & \|A u(t)\|^{2}+\|\ddot{u}(s)-f(s)\|^{2}+\gamma^{2}\|\dot{u}(s)\|^{2}+\gamma \frac{d}{d s}\|\dot{u}(s)\|^{2} \\
& -2 \gamma\langle\dot{u}(s), f(s)\rangle+2\langle A u(t), \ddot{u}(s)+\gamma \dot{u}(s)-f(s)\rangle .
\end{aligned}
$$

Therefore

$$
\begin{aligned}
\frac{1}{t_{k}} \int_{0}^{t_{k}}\left(\|\dot{u}(s)\|^{2}-\alpha\|A u(t)-A u(s)\|^{2}\right) d s & =\frac{1}{t_{k}} \int_{0}^{t_{k}}\left(\left(1-\alpha \gamma^{2}\right)\|\dot{u}(s)\|^{2}-\alpha\|A u(t)\|^{2}-\alpha\|\ddot{u}(s)-f(s)\|^{2}\right) d s \\
& -\frac{\alpha \gamma}{t_{k}}\left(\left\|\dot{u}\left(t_{k}\right)\right\|^{2}-\|\dot{u}(0)\|^{2}\right)+\frac{2 \alpha \gamma}{t_{k}} \int_{0}^{t_{k}}\langle\dot{u}(s), f(s)\rangle d s \\
& -\frac{2 \alpha}{t_{k}}\left\langle A u(t), \dot{u}\left(t_{k}\right)+\gamma u\left(t_{k}\right)-(\dot{u}(0)+\gamma u(0))-\int_{0}^{t_{k}} f(s) d s\right\rangle .
\end{aligned}
$$

Now, since $\alpha \gamma^{2}>1, f \in L^{1}$, and $u$ and $\dot{u}$ are bounded, by taking the lim sup as $k \rightarrow+\infty$, we get

$$
\limsup _{k \rightarrow+\infty} \frac{1}{t_{k}} \int_{0}^{t_{k}}\left(\|\dot{u}(s)\|^{2}-\alpha\|A u(t)-A u(s)\|^{2}\right) d s \leq-\alpha\|A u(t)\|^{2} .
$$

Hence

$$
\langle A u(t), u(t)-p\rangle \geq \alpha\|A u(t)\|^{2} .
$$

Multiplying (1.1) by $u(t)-p$ and using (2.1), we get

$$
\ddot{h_{p}}(t)-\|\dot{u}(t)\|^{2}+\gamma \dot{h_{p}}(t)+\alpha\|A u(t)\|^{2} \leq\langle f(t), u(t)-p\rangle .
$$

where $h_{p}(t):=\frac{1}{2}\|u(t)-p\|^{2}$. Substituting from (1.1), yields

$$
\ddot{h_{p}}(t)+\gamma \dot{h_{p}}(t)+\left(\alpha \gamma^{2}-1\right)\|\dot{u}(t)\|^{2}+\alpha\|\ddot{u}(t)-f(t)\|^{2}+\alpha \gamma \frac{d}{d t}\|\dot{u}(t)\|^{2} \leq M_{2}\|f(t)\|,
$$

where $M_{2}:=\sup _{t \geq 0}\|2 \alpha \gamma \dot{u}(t)+u(t)-p\|$. Integrating the above inequality on the interval $[0, t]$, we get:

$$
\dot{h_{p}}(t)+\gamma h_{p}(t)+\left(\alpha \gamma^{2}-1\right) \int_{0}^{t}\|\dot{u}(\tau)\|^{2} d \tau+\alpha \int_{0}^{t}\|\ddot{u}(\tau)-f(\tau)\|^{2} d \tau+\alpha \gamma\|\dot{u}(t)\|^{2} \leq M_{2} \int_{0}^{t}\|f(\tau)\| d \tau+C,
$$

where $C$ is some constant. The above inequality implies that $\dot{u}, \ddot{u}-f \in L^{2}(0,+\infty ; H)$, which yields $A u \in$ $L^{2}(0,+\infty ; H)$. Since $A$, as well as $u$ are Lipschitz (because $\dot{u}$ is bounded), we deduce that $A u$ is Lipschitz too. Therefore $A u(t) \rightarrow 0$ as $t \rightarrow+\infty$. Using the maximality of $A$, we conclude that every weak cluster point of $u(t)$ is in $A^{-1}(0)$. Hence $A^{-1}(0) \neq \emptyset$. Now, let $q$ be a weak cluster point of $u(t)$. Multiplying (1.1) by $u(t)-q$ and using the monotonicity of $A$, we get

$$
\ddot{h_{q}}(t)+\gamma \dot{h_{q}}(t) \leq M_{3}\|f(t)\|+\|\dot{u}(t)\|^{2},
$$


where $h_{q}(t):=\frac{1}{2}\|u(t)-q\|^{2}$ and $M_{3}:=\sup _{t \geq 0}\|u(t)-q\|$. Applying Lemma 2.1 yields that $\lim _{t \rightarrow+\infty}\|u(t)-q\|^{2}$ exists for each weak cluster point $q$ of $u(t)$. Let $q_{1}$ and $q_{2}$ be two weak cluster points of $u(t)$. Then $\lim _{t \rightarrow+\infty}(\| u(t)-$ $\left.q_{1}\left\|^{2}-\right\| u(t)-q_{2} \|^{2}\right)$ exists. It follows that $\lim _{t \rightarrow+\infty}\left\langle u(t), q_{1} q_{2}\right\rangle$ exists. Therefore $\left\langle q_{1}, q_{1}-q_{2}\right\rangle=\left\langle q_{2}, q_{1}-q_{2}\right\rangle$, and so $q_{1}=q_{2}$. This shows that $u(t)$ converges weakly to some point in $A^{-1}(0)$. Let $u(t) \rightarrow q$. For an arbitrary $x \in H$, we have

$$
\|u(t)-q\|^{2}=\|u(t)-x\|^{2}+\|x-q\|^{2}+2\langle u(t)-x, x-q\rangle,
$$

and therefore

$$
\lim _{t \rightarrow+\infty}\|u(t)-q\|^{2}=\limsup _{t \rightarrow+\infty}\|u(t)-x\|^{2}-\|x-q\|^{2} .
$$

The above identity shows that $q$ is the asymptotic center of $u(t)$.

In the following theorem we provide a sufficient condition for the strong convergence of the solutions to (1.1).

Theorem 2.3. Let $A$ be an $\alpha$-inverse strongly monotone and $\beta$-strongly monotone operator. If $f \in L^{1}(0,+\infty ; H)$, $\alpha \gamma^{2}>1$ and $u(t)$ satisfy (1.1), then $u(t)$ converges strongly to some $q \in A^{-1}(0)$, which is also the asymptotic center of $u(t)$, if and only if $u(t)$ is bounded.

Proof. Necessity is clear. For the sufficiency, we note that from Theorem 2.2, there exists some $q \in A^{-1}(0)$ such that $u(t) \rightarrow q$. Now the strong monotonicity of $A$ implies

$$
\beta\|u(t)-q\|^{2} \leq\langle f(t)-\ddot{u}(t)-\gamma \dot{u}(t), u(t)-q\rangle \leq M\|f(t)\|+\|\dot{u}(t)\|^{2}-\ddot{h_{q}}(t)-\gamma \dot{h_{q}}(t),
$$

where $M:=\sup _{t \geq 0}\|u(t)-q\|$ and $h_{q}(t):=\frac{1}{2}\|u(t)-q\|^{2}$. Integrating the above inequality on the interval [0, $t$, we get:

$$
\beta \int_{0}^{t}\|u(s)-q\|^{2} d s \leq M \int_{0}^{t}\|f(s)\| d s+\int_{0}^{t}\|\dot{u}(s)\|^{2} d s-\dot{h_{q}}(t)-\gamma h_{q}(t)+C,
$$

where $C$ is some constant. Since $f \in L^{1}(0,+\infty ; H)$, and by the proof of Theorem $2.2, \dot{u} \in L^{2}(0,+\infty ; H)$ and $\dot{h_{q}}$ is bounded, therefore $\|u(t)-q\| \in L^{2}(0,+\infty ; H)$. Again, from the proof of Theorem 2.2, we know that $\lim _{t \rightarrow+\infty}\|u(t)-q\|$ exists and this concludes the proof.

Remark 2.4. We note that our assumptions on the operator $A$ contains the case where $A=I-T$, with $T$ nonexpansive (that is $\|T x-T y\| \leq\|x-y\|$, for every $x, y \in H$ ).

\section{Inexact inertial proximal method}

In [9], Alvarez and Attouch studied the iterative method

$$
u_{n+1}-u_{n}-\alpha_{n}\left(u_{n}-u_{n-1}\right)+\lambda_{n} A u_{n+1} \ni 0,
$$

where $A$ is a general maximal monotone operator. Assuming that $A^{-1}(0) \neq \emptyset$, and with the following conditions on the parameters

(i) $\exists \lambda>0$ such that $\forall n \in \mathbb{N}, \lambda_{n} \geq \lambda$,

(ii) $\exists \alpha \in[0,1)$ such that $\forall n \in \mathbb{N}, 0 \leq \alpha_{n} \leq \alpha$,

(iii) $\sum_{n=1}^{+\infty} \alpha_{n}\left\|u_{k}-u_{k-1}\right\|^{2}<+\infty$,

they obtained the weak convergence of $u_{n}$. At the end of their paper, they raised the open problem to find convergence results for the inexact inertial proximal method, as well as to develop a general theory to guide 
the choices of the parameters $\lambda_{n}$ and $\alpha_{n}$. In this section, we consider the following discrete counterpart of (1.1) which is also the inexact version of (3.1)

$$
u_{n+1}-u_{n}-\alpha_{n}\left(u_{n}-u_{n-1}\right)+\lambda_{n} A u_{n+1} \ni f_{n},
$$

where $A$ is a general maximal monotone operator. Motivated by [11], we note that by taking $\alpha_{n}\left(u_{n}-u_{n-1}\right)+f_{n}$ as the nonhomogeneous term, the inexact inertial proximal algorithm (3.2) is reduced to the inexact proximal point algorithm

$$
u_{n+1}-u_{n}+\lambda_{n} A u_{n+1} \ni g_{n},
$$

where $g_{n}:=\alpha_{n}\left(u_{n}-u_{n-1}\right)+f_{n}$. Therefore, if $u_{n}$ given by (3.2) is bounded, then by using the results in [11], we prove ergodic, as well as convergence theorems for the sequence $u_{n}$ satisfying (3.2), and provide an answer to the open problem raised in [9], without assuming $A^{-1}(0) \neq \emptyset$. Set

$$
w_{n}:=\left(\sum_{k=1}^{n} \lambda_{k}\right)^{-1}\left(\sum_{k=1}^{n} \lambda_{k} u_{k+1}\right) .
$$

We denote by $\omega_{w}\left(w_{n}\right)$ the set of all weak cluster points of the sequence $w_{n}$. First, we state the following lemma from classical analysis.

Lemma 3.1. Suppose that $a_{n}$ and $b_{n}$ are nonnegative real sequences and that $\sum_{n=1}^{+\infty} b_{n}<+\infty$. If

$$
a_{n+1} \leq a_{n}+b_{n}, \quad \forall n \geq 1,
$$

then there exists $\lim _{n \rightarrow+\infty} a_{n}$.

The following is a weak ergodic theorem for the sequence $u_{n}$.

Theorem 3.2. Assume that $u_{n}$, given by (3.2) is bounded and (i) and (ii) bellow hold:

(i) $\sum_{n=1}^{+\infty} \lambda_{n}=+\infty$,

(ii) $\sum_{n=1}^{+\infty}\left\|\alpha_{n}\left(u_{n}-u_{n-1}\right)+f_{n}\right\|<+\infty$.

Then the sequence $w_{n}$ converges weakly to some $p \in A^{-1}(0)$, which is also the asymptotic center of the sequence $u_{n}$.

Proof. By the monotonicity of $A$, for all $k, n \in \mathbb{N}$, we have

$$
\left\langle A u_{k+1}, u_{n+1}\right\rangle+\left\langle A u_{n+1}, u_{k+1}\right\rangle \leq\left\langle A u_{n+1}, u_{n+1}\right\rangle+\left\langle A u_{k+1}, u_{k+1}\right\rangle .
$$

Multiplying both sides of the above inequality by $\lambda_{k} \lambda_{n}$, then substituting $\lambda_{n} A u_{n+1}$ and $\lambda_{k} A u_{k+1}$ from (3.2), we get:

$$
\begin{aligned}
\left\langle u_{k}-u_{k+1}+\alpha_{k}\left(u_{k}-u_{k-1}\right)\right. & \left.+f_{k}, \lambda_{n} u_{n+1}\right\rangle+\left\langle u_{n}-u_{n+1}+\alpha_{n}\left(u_{n}-u_{n-1}\right)+f_{n}, \lambda_{k} u_{k+1}\right\rangle \\
\leq & \left\langle u_{n}-u_{n+1}+\alpha_{n}\left(u_{n}-u_{n-1}\right)+f_{n}, \lambda_{k} u_{n+1}\right\rangle+\left\langle u_{k}-u_{k+1}+\alpha_{k}\left(u_{k}-u_{k-1}\right)+f_{k}, \lambda_{n} u_{k+1}\right\rangle .
\end{aligned}
$$

Since $u_{n}$ is bounded, so is $w_{n}$, and therefore $\omega_{w}\left(w_{n}\right) \neq \emptyset$. Let $p \in \omega_{w}\left(w_{n}\right)$. Then there exists a subsequence $m_{j}$ such that $w_{m_{j}} \rightarrow p$. Summing up both sides of the above inequality from $k=1$ to $m_{j}$, dividing by $\sum_{k=1}^{m_{j}} \lambda_{k}$, and then taking the limit as $j \rightarrow+\infty$, we get:

$$
\left\langle u_{n}-u_{n+1}+\alpha_{n}\left(u_{n}-u_{n-1}\right)+f_{n}, u_{n+1}-p\right\rangle \geq 0 .
$$

It follows that:

$$
\left\|u_{n+1}-p\right\| \leq\left\|u_{n}-p\right\|+\left\|\alpha_{n}\left(u_{n}-u_{n-1}\right)+f_{n}\right\| .
$$

Applying Lemma 3.1, we get $\lim _{n \rightarrow+\infty}\left\|u_{n}-p\right\|$ exists. Hence for any $p, q \in \omega_{w}\left(w_{n}\right), \lim _{n \rightarrow+\infty} \frac{1}{2}\left(\left\|u_{n}-p\right\|^{2}-\| u_{n}-\right.$ $q \|^{2}$ ) and therefore $\lim _{n \rightarrow+\infty}\left\langle u_{n}, p-q\right\rangle$ exist. This implies that $\langle q, p-q\rangle=\langle p, p-q\rangle$, and hence $p=q$. Therefore 
$w_{n} \rightarrow p$. Now, we are going to show that $p \in A^{-1}(0)$, which implicitly implies that $A^{-1}(0)$ is nonempty. For this, let $x \in D(A)$ and $y \in A x$. Then

$$
\begin{aligned}
\left\langle y, x-w_{n}\right\rangle & =\left(\sum_{k=1}^{n} \lambda_{k}\right)^{-1} \sum_{k=1}^{n} \lambda_{k}\left\langle y, x-u_{k+1}\right\rangle \\
& =\left(\sum_{k=1}^{n} \lambda_{k}\right)^{-1} \sum_{k=1}^{n} \lambda_{k}\left(\left\langle y-A u_{k+1}, x-u_{k+1}\right\rangle+\left\langle A u_{k+1}, x-u_{k+1}\right\rangle\right) \\
& \geq\left(\sum_{k=1}^{n} \lambda_{k}\right)^{-1} \sum_{k=1}^{n}\left\langle u_{k} u_{k+1}+\alpha_{k}\left(u_{k} u_{k 1}\right)+f_{k}, x-u_{k+1}\right\rangle \\
& \geq\left(\sum_{k=1}^{n} \lambda_{k}\right)^{-1} \sum_{k=1}^{n}\left(\frac{1}{2}\left\|u_{k+1}-x\right\|^{2}-\frac{1}{2}\left\|u_{k}-x\right\|^{2}-\left\|\alpha_{k}\left(u_{k}-u_{k-1}\right)+f_{k}\right\|\left\|u_{k+1}-x\right\|\right) \\
& \geq \frac{-\left(\sum_{k=1}^{n} \lambda_{k}\right)^{-1}}{2}\left\|u_{1}-x\right\|^{2}-\left(\sum_{k=1}^{n} \lambda_{k}\right)^{-1} \sum_{k=1}^{n}\left\|\alpha_{k}\left(u_{k}-u_{k-1}\right)+f_{k}\right\|\left\|u_{k+1}-x\right\| .
\end{aligned}
$$

Letting $n \rightarrow+\infty$, this yields $\langle y, x-p\rangle \geq 0$. Now the maximality of $A$ implies that $p \in A^{-1}(0)$. To prove that $p$ is the asymptotic center of the sequence $u_{n}$, choose an arbitrary element $x \in H$. Then we have

$$
\left\|u_{n+1}-p\right\|^{2}=\left\|u_{n+1}-x\right\|^{2}-\|x\|^{2}+\|p\|^{2}-2\left\langle u_{n+1}, p-x\right\rangle .
$$

Multiplying the above identity by $\lambda_{n}$, summing up from $n=1$ to $n=N$, dividing by $\sum_{n=1}^{N} \lambda_{n}$, and then letting $N \rightarrow+\infty$, we get:

$$
\lim _{n \rightarrow+\infty}\left\|u_{n}-p\right\|^{2} \leq \limsup _{n \rightarrow+\infty}\left\|u_{n}-x\right\|^{2}-\|x-p\|^{2}<\limsup _{n \rightarrow+\infty}\left\|u_{n}-x\right\|^{2},
$$

if $x \neq p$, and this concludes the result.

Remark 3.3. By [12, Remark 14], the weak (resp.strong) convergence of $u_{n}$ given by (3.3) (and so $u_{n}$ given by (3.2)) in the case $g_{n}\left(=\alpha_{n}\left(u_{n}-u_{n-1}\right)+f_{n}\right) \equiv 0$ implies the weak (resp. strong) convergence of $u_{n}$ in the case where $g_{n} \neq 0$, provided that $\sum_{n=1}^{+\infty}\left\|g_{n}\right\|<+\infty$. Therefore for the study of weak (resp. strong) convergence of $u_{n}$ given by (3.2), we may assume without loss of generality that $\alpha_{n}\left(u_{n}-u_{n-1}\right)+f_{n} \equiv 0$. We note that in this case the inexact inertial proximal algorithm (3.2) is reduced to the proximal point algorithm.

Theorem 3.4. Let $u_{n}$ be the sequence given by (3.2). Assume that the following conditions hold:

(i) $\sum_{n=1}^{+\infty} \lambda_{n}^{2}=+\infty$,

(ii) $\sum_{n=1}^{+\infty}\left\|\alpha_{n}\left(u_{n}-u_{n-1}\right)+f_{n}\right\|<+\infty$.

Then the sequence $u_{n}$ converges weakly to some $p \in A^{-1}(0)$, which is also the asymptotic center of $u_{n}$, if and only if $\lim \inf _{n \rightarrow+\infty}\left\|w_{n}\right\|<+\infty$.

Proof. Necessity is clear. To prove the sufficiency, by Remark 3.3, it is enough to consider the case where $\alpha_{n}\left(u_{n}-u_{n-1}\right)+f_{n} \equiv 0$. Let $p$ be a weak cluster point of $w_{n}$. From (3.2) we have $u_{n}-u_{n+1} \in \lambda_{n} A u_{n+1}$, and so

$$
\begin{aligned}
\lambda_{n}^{2}\left\|A u_{n+1}\right\|^{2} & =\left\|u_{n}-p\right\|^{2}-\left\|u_{n+1}-p\right\|^{2}-2\left\langle u_{n}-u_{n+1}, u_{n+1}-p\right\rangle \\
& \leq\left\|u_{n}-p\right\|^{2}-\left\|u_{n+1}-p\right\|^{2},
\end{aligned}
$$

where in the last inequality we used (3.4), since (i) implies that $\sum_{n=1}^{+\infty} \lambda_{n}=+\infty$. As we have already seen in the proof of Theorem 3.2, $\lim _{n \rightarrow+\infty}\left\|u_{n}-p\right\|^{2}$ exists. Summing up both sides of the above inequality from $n=1$ to $N$, and letting $N \rightarrow+\infty$, we get:

$$
\sum_{n=1}^{+\infty} \lambda_{n}^{2}\left\|A u_{n+1}\right\|^{2} \leq\left\|u_{1}-p\right\|^{2}<+\infty
$$


Condition (i) implies that $\liminf _{n \rightarrow+\infty}\left\|A u_{n}\right\|^{2}=0$. By using the monotonicity of $A$ and (3.3) with $g_{n} \equiv 0$, we get:

$$
\left\langle A u_{n}-A u_{n+1}, \lambda_{n} A u_{n+1}\right\rangle \geq 0,
$$

and therefore

$$
\left\|A u_{n+1}\right\| \leq\left\|A u_{n}\right\| .
$$

Hence $\lim _{n \rightarrow+\infty}\left\|A u_{n}\right\|=0$. Assume that $u_{n_{j}} \rightarrow q$. By the monotonicity of $A$, we have

$$
\left\langle A u_{n+1}-A u_{n_{j}}, u_{n+1}-u_{n_{j}}\right\rangle \geq 0 .
$$

Letting $j \rightarrow+\infty$ in the above inequality, we get

$$
\left\langle A u_{n+1}, u_{n+1}-q\right\rangle \geq 0 .
$$

Substituting $A u_{n+1}$ from (3.3) with $g_{n} \equiv 0$, yields

$$
\left\|u_{n+1}-q\right\| \leq\left\|u_{n}-q\right\| .
$$

Therefore $\lim _{n \rightarrow+\infty}\left\|u_{n}-q\right\|^{2}$ exists. Now the result follows by applying the same argument as in the proof of Theorem 3.2.

In the next two theorems we give sufficient conditions for the strong convergence of $u_{n}$.

Theorem 3.5. Assume that $(I+A)^{-1}$ is compact, and the conditions (i) and (ii) in Theorem 3.4 hold. Then the sequence $u_{n}$ given by (3.2) converges strongly to an element $p \in A^{-1}(0)$, which is also the asymptotic center of $u_{n}$, if and only liminf $\operatorname{in}_{n \rightarrow+\infty}\left\|w_{n}\right\|<+\infty$.

Proof. Necessity is clear. To prove the sufficiency by Remark 3.3, it is enough to consider the case $\alpha_{n}\left(u_{n}-\right.$ $\left.u_{n-1}\right)+f_{n} \equiv 0$. The proof of Theorem 3.4 implies that $\lim _{n \rightarrow+\infty}\left\|A u_{n}\right\|=0$, and $u_{n} \rightarrow p$. Therefore $(I+A) u_{n}$ is bounded. Since $(I+A)^{-1}$ is compact, then $u_{n}$ contains a strongly convergent subsequence to some $p \in H$, say $u_{n_{j}}$. From the monotonicity of $A$, we have

$$
\left\langle A u_{n}-A u_{n_{j}}, u_{n}-u_{n_{j}}\right\rangle \geq 0,
$$

and therefore by letting $j \rightarrow+\infty$, we get

$$
\left\langle A u_{n}, u_{n}-p\right\rangle \geq 0 \text {. }
$$

Now the same argument as in the proof of Theorem 3.4 yields that $\lim _{n \rightarrow+\infty}\left\|u_{n}-p\right\|^{2}$ exists, which concludes the proof.

Theorem 3.6. Suppose that $A$ is strongly monotone and the conditions (i) and (ii) in Theorem 3.2 hold. Then the sequence $u_{n}$ given by (3.2) converges strongly to an element $p \in A^{-1}(0)$, which is also the asymptotic center of $u_{n}$, if and only if $\lim _{\inf }{ }_{n \rightarrow+\infty}\left\|w_{n}\right\|<+\infty$.

Proof. Necessity is clear, and we only need to prove the sufficiency. By Theorem 3.2, there exists some $p \in$ $A^{-1}(0)$ such that $w_{n} \rightarrow p$, and $\lim _{n \rightarrow+\infty}\left\|u_{n}-p\right\|^{2}$ exists. The strong monotonicity of $A$ implies that there is an $\alpha>0$ such that

$$
\alpha \lambda_{n}\left\|u_{n+1}-p\right\|^{2} \leq\left\langle\lambda_{n} A u_{n+1}, u_{n+1}-p\right\rangle .
$$

Substituting from (3.2) in the above inequality, we get:

$$
\begin{aligned}
\alpha \lambda_{n}\left\|u_{n+1}-p\right\|^{2} & \leq\left\langle u_{n}-u_{n+1}+\alpha_{n}\left(u_{n}-u_{n-1}\right)+f_{n}, u_{n+1}-p\right\rangle \\
& \leq \frac{1}{2}\left\|u_{n}-p\right\|^{2}-\frac{1}{2}\left\|u_{n+1}-p\right\|^{2}+M\left\|\alpha_{n}\left(u_{n}-u_{n-1}\right)+f_{n}\right\|,
\end{aligned}
$$


where $M:=\sup _{n \geq 1}\left\|u_{n}-p\right\|$. By summing up both sides of the above inequality from $n=1$ to $N$, and letting $N \rightarrow+\infty$, we get:

$$
\sum_{n=1}^{+\infty} \lambda_{n}\left\|u_{n+1}-p\right\|^{2}<+\infty
$$

Since $\sum_{n=1}^{+\infty} \lambda_{n}=+\infty$, (3.5) implies that $\lim _{\inf _{n \rightarrow+\infty}}\left\|u_{n}-p\right\|^{2}=0$, which concludes the proof.

Remark 3.7. Assuming that $\alpha_{n}, f_{n} \in l^{1}$, the condition (ii) in Theorems 3.2 and 3.4 is satisfied, if $u_{n}$ is bounded.

\section{Conclusions}

In this paper, we studied the weak and strong convergence to a zero of the operator for the solutions to the nonlinear oscillator with damping with a maximal monotone and inverse strongly monotone operator $A$, without assuming the zero set of $A$ to be nonempty. In particular, we showed that the zero set of $A$ is nonempty if and only if bounded solutions exist. We also studied and proved similar results for the asymptotic behavior of the inexact inertial proximal algorithm obtained by its discretization, where the operator $A$ is only assumed to be maximal monotone, without assuming its zero set to be nonempty, therefore extending and solving an open problem raised in [9]. As a future direction for research, it might be interesting to explore and extend these ideas to more general operators and settings, and with nonconstant damping.

Acknowledgments: This research is done while the second author was visiting the University of Texas at El Paso. This visit is funded partially by the Iran Ministry of Science, Research and Technology. The second author would like to thank Professor Djafari Rouhani and the Department of Mathematical Sciences for their hospitality at the University of Texas at El Paso during his visit.

\section{References}

[1] Djafari Rouhani B., Asymptotic behaviour of quasi-autonomous dissipative systems in Hilbert spaces, J. Math. Anal. Appl., $1990,147,465-476$

[2] Djafari Rouhani B., Asymptotic behaviour of almost nonexpansive sequences in a Hilbert space, J. Math. Anal. Appl., 1990, 151, 226-235

[3] Djafari Rouhani B., Khatibzadeh H., Asymptotic behavior of bounded solutions to some second order evolution systems, Rocky Mountain J. Math., 2010, 40, 1289-1311

[4] Djafari Rouhani B., Khatibzadeh H., A strong convergence theorem for solutions to a nonhomogeneous second order evolution equation, J. Math. Anal. Appl., 2010, 363, 648-654

[5] Djafari Rouhani B., Khatibzadeh H., Asymptotic behavior of bounded solutions to a class of second order nonhomogeneous evolution equations, Nonlinear Anal., 2009, 70, 4369-4376

[6] Alvarez F., On the minimizing property of a second order dissipative system in Hilbert spaces, SIAM J. Control Optim., 2000, 38, 1102-1119

[7] Attouch H., Goudou X., Redont P., The heavy ball with friction method, I. The continuous dynamical system: global exploration of the local minima of real-valued function by asymptotic analysis of a dissipative dynamical system, Commun. Contemp. Math., 2000, 2, 1-34

[8] Attouch H., Maingé P. E., Asymptotic behavior of second-order dissipative evolution equations combining potential with non-potential effects, ESAIM Control Optim. Calc. Var., 2011, 17, 836-857

[9] Alvarez F., Attouch H., An inertial proximal method for maximal monotone operators via dicretization of a nonlinear oscillator with damping, Set Valued Anal., 2001, 9, 3-11

[10] Edelstein M., The construction of an asymptotic center with a fixed-point property, Bull. Amer. Math. Soc., 1972, 78, 206208

[11] Djafari Rouhani B., Khatibzadeh H., On the proximal point algorithm, J. Optim. Theory Appl., 2008, 137, 411-417

[12] Brézis H., Lions P. L., Produits infinis de résolvantes, Israel J. Math., 1978, 29, 329-345 